\title{
Manipulating single second mode transparency in a corrugated waveguide via the thickness of sputtered gold
}

\author{
Dan Xu, Ya-Xian Fan*, Tang-Qing Sang, Lan-Lan Xu, Aysha Bibi, \\ Zhi-Yong Tao** \\ Key Lab of In-fiber Integrated Optics, Ministry Education of China, Harbin Engineering \\ University, Harbin 150001, People's Republic of China. \\ Photonics Research Center, College of Science, Harbin Engineering University, Harbin \\ 150001, People's Republic of China.
}

\begin{abstract}
We propose a classical analog of electromagnetically induced transparency in a cylindrical waveguide with undulated metallic walls. The transparency, induced by multi-mode interactions in waveguides, not only has a narrow line-width, but also consists of a single second-order transverse mode, which corresponds to the Bessel function distributions investigated extensively due to their unique characteristics. By increasing the thickness of sputtered gold layers of the waveguide, we demonstrate a frequency-agile single mode transparency phenomenon in a terahertz radiation. It is found that the center frequency of the transparency is linearly related to the gold thickness, indicating the achievement of a controllable single mode terahertz device. The field distributions at the cross-sections of outlets verify the single second mode transparency and indicate the mechanism of its frequency manipulation, which will significantly benefit the mode-control engineering in terahertz applications.
\end{abstract}

Keywords: Transparency, Single mode, Mode interaction, Frequency manipulation

PACS: 42.25.Bs, 42.82.Et, 41.20.Jb

\footnotetext{
* Corresponding author.

** Principal corresponding author.

Email addresses: yxfan@hrbeu.edu.cn (Ya-Xian Fan), zytao@hrbeu.edu.cn (Zhi-Yong Tao)
} 


\section{Introduction}

Electromagnetically induced transparency (EIT) has been paid extensive attention $[1,2]$ since it was used by Harris et al. to investigate the nonlinear optical phenomenon [3]. Quantum interference induced by two laser beams results in an opaque medium to be transparent in an extremely narrow spectrum, that can not only enhance nonlinear effects but also significantly slow the light. Since the transparency is very promising in various applications such as dispersion engineering, quantum or optical information storage, and slow light, the EIT-like behavior have been considerably demonstrated in classical systems in recent years. Totsuka et al. have employed two microspheres with different diameters to induce a EIT-like transparency and have observed slow light [4]. Papasimakis et al. have investigated a metamaterial analog of EIT [5], in which electromagnetic pulses have been delayed by a subwavelength fish-scale planar grating. Later, the plasmon-induced transparency has also been investigated to slow down the light [6]. Moreover, other transparencies induced by Fano-like resonances [7] and by coupling of Tamm and defect states [8] have been studied in a photonic circuit and in a terahertz $(\mathrm{THz})$ plasmonic crystals, respectively. Almost all the high quality EIT-like behaviors have been realized by appending the micro resonators to a straight waveguide, but no high-order modes were discussed.

It is worth noting that the high-order modes will participate in resonances and play a major role when the transverse scale of waveguides gets larger. In 1998, Pogrebnyak has investigated the electromagnetic properties of a corrugated waveguide and found the miniband behavior of the spectrum caused by the resonances of transverse modes [9]. The THz non-Bragg gaps [10] has been investigated in the periodic metallic waveguides, indicating the fundamental role of the high-order modes in wave-structure interactions. Especially, the transverse mode in a cylindrical waveguide always associates with a pure Bessel function distribution. These Bessel beams have found applications in various fields such as optical tweezers, optically bound control, virtual ghost imaging, and nanofabrications [11, 12, 13, 14].

On the other hand, $\mathrm{THz}$ radiation in a frequency range between the infrared and microwaves has been paid more attention owing to its applications in communications, imaging, non-destructive evaluation, spectroscopy biochemical sensing, security screening, and so on [15]. Besides the low-loss and low-dispersion waveguide structures, the single mode operation in $\mathrm{THz}$ frequency range has also been investigated. By introducing microstructured 
air holes, a directional coupler at $1 \mathrm{THz}$ was optimized with single mode operation last year [16]. Mitrofanov and Harrington have detected four different $\mathrm{THz}$ modes in a dielectric-lined cylindrical metallic waveguide by their different time delays at the output [17] and Tang et al. have investigated the dependence of mode structure on the ellipticity in a silver coated elliptical hollow fiber [18]. The single $\mathrm{THz}$ modes have also been produced and used in superluminal devices [19], quantum cascade lasers [20], nondiffractive beams [21], low propagation loss and low bending loss waveguides [22].

Therefore, it is very intriguing to realize and manipulate a transparency of single high-order modes in a cylindrical $\mathrm{THz}$ waveguide. Here, we show that the phenomenon occurs as a result of multi-mode interactions, which can be interpreted by a graph shifting method. When the material for manufacturing the waveguide is considered to be $\mathrm{Au}$, the transparent frequency will shift according to the thickness of sputtered gold layers. Then, we demonstrate the transparency characteristics when the thickness increases, including the band width, the transmittance, the slow down factor, the propagation length, and the transverse distributions of the electromagnetic fields. Finally, a terahertz single mode transparency manipulated by the gold thickness is discussed and its mechanism is revealed.

\section{Multi-mode interactions}

In order to transmit a single high-order mode in a waveguide, we break the orthogonality of the eigenmodes of a smooth waveguide and manipulate the energy redistribution between them by means of undulating the tube walls. The geometry structure is shown in Fig. 1 (a), where $\Lambda$ and $h$ are the period and the height of wall undulations, respectively. $t$, the thickness of gold layers, is chosen as zero in this section for the convenience of multimode analysis. It is found that a 10-period long hollow tube can produce the proposed transparency very well. According to the Floquet theorem and dispersion relations $[23,24]$, we can obtain the relationship between the frequency $f$ and the reference propagation constant $\beta$ for the $m$ th order mode

of the $n$th spatial harmonic:

$$
f=\frac{c}{2 \pi} \sqrt{\left(\frac{k_{r}^{(m)}}{r_{0}}\right)^{2}+\left(\frac{2 n \pi}{\Lambda}+\beta\right)^{2}}
$$



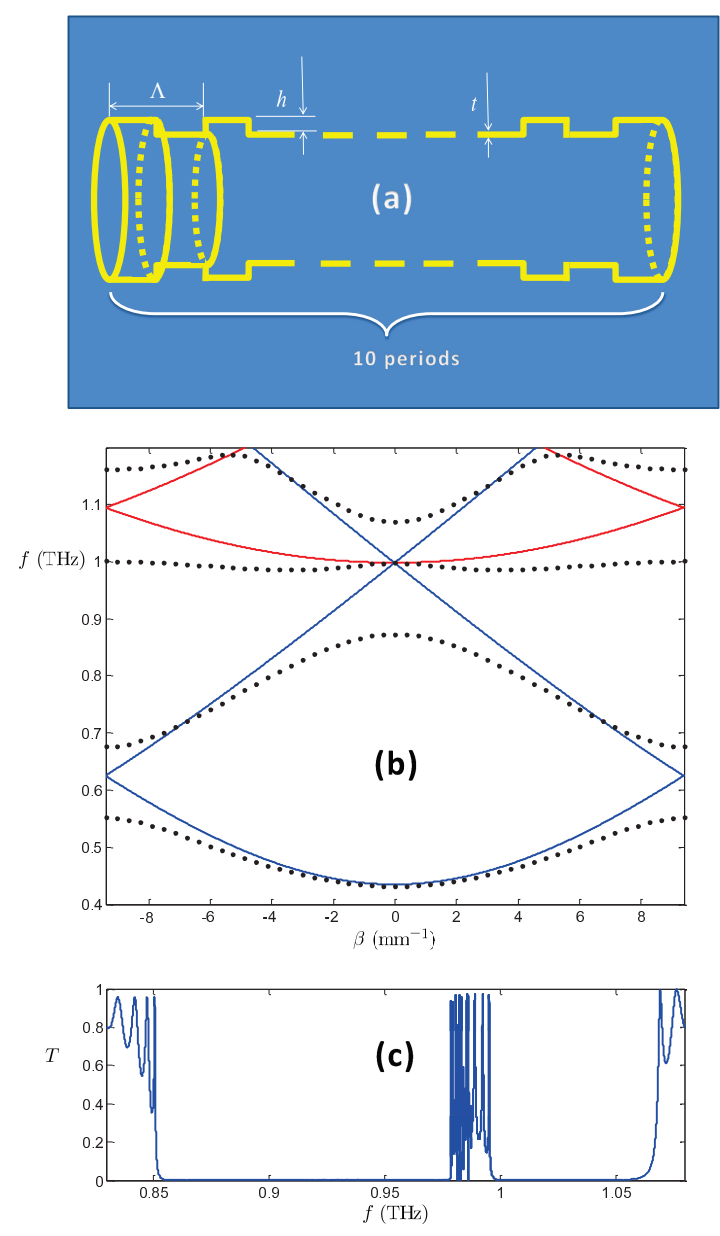

Figure 1: Geometry parameters and band structures of a gold tube transparent to a single second mode. (a) A 10-period gold tube with corrugated walls is designed to realize the single second mode transparency. The thickness of the sputtered gold layer $t$ is increased to the inside to modulate the frequency of the transparency. (b) The blue and red lines denote the first and second transverse modes, respectively. Mode manipulation using the shifting method of reference lines results in the three mode interactions near $1 \mathrm{THz}$ when the period and the mean radius of the waveguide are selected as $334 \mu \mathrm{m}$ and 264 $\mu \mathrm{m}$, respectively. The bold dots constitute dispersion curves confirming the manipulation. (c) The transmission simulated by the finite element method with the perfect electrical boundary conditions when $t=0 \mu \mathrm{m}$. 
where $r_{0}$ is the mean radius and $k_{r}^{(m)}$ is the zeros of the zeroth-order Bessel functions for transverse magnetic (TM) waves. For the first and second

modes, $k_{r}^{(m)}$ is equal to 2.4048 and 5.5201, respectively. Thus, the reference lines defined by Eq. (1) for the first two modes in the Brillouin zone are illustrated in Fig. 1 (b) by the blue and red solid lines, respectively. In a graph shifting method [23], changing the period and mean radius of the waveguide always results in the vertical movements of solid lines in the first Brillouin zone and the relative location of the red and blue lines varies to achieve different spectrum structures. Setting $\Lambda=334 \mu \mathrm{m}$ and $r_{0}=264 \mu \mathrm{m}$, we can obtain a three mode interaction at $1 \mathrm{THz}$ where a red line (the second mode) intersects with two blue lines (the first modes). A single high-order mode transparency could be expected. By using the numerical method in [24], we calculated the dispersion curves of the structure with $\Lambda=334 \mu \mathrm{m}$, $r_{0}=264 \mu \mathrm{m}$, and $h=52.8 \mu \mathrm{m}$. The results are depicted in Fig. 1 (b) by the bold dots, indicating that the two lower modes are pushed away and a single second mode is left. It is clear that at $1 \mathrm{THz}$, there is a narrow pass band in a wide stop band related to the second mode. The solid line in Fig. 1 (c) is the transmission $T$ simulated by COMSOL Multiphysics with the finite element method. The perfect electrical boundary conditions were assigned to the tube wall when the gold thickness $t=0 \mu \mathrm{m}$. The simulated result confirms the transparency in a stop band.

\section{Frequency shifting}

To manipulate the frequency of the transparency, we have made the tube wall with different thicknesses of gold layers. With the fixed values of the period $\Lambda$, of the height $h$, and of the mean outer radius $r_{0}$, we obtained different waveguides with the gold layers thickened to the inside. Around $1 \mathrm{THz}$, the Drude model was employed in the simulations for the dielectric function of $\mathrm{Au}$ with

$$
\varepsilon=1-\frac{\omega_{p}^{2}}{\omega^{2}+\mathrm{i} \omega \omega_{\tau}}
$$

where the circular frequency $\omega=2 \pi f$, the plasma frequency $\omega_{p}=1.37 \times 10^{4}$ $\mathrm{THz}$, and the damping frequency $\omega_{\tau}=40.7 \mathrm{THz}$ [25]. The transmissions of different tubes are presented in Fig. 2 (a) when the inner gold thickness $t$ varies from $10 \mu \mathrm{m}$ to $70 \mu \mathrm{m}$. We can find out the transparencies in the stop bands for seven different $\mathrm{THz}$ waveguides. When the thickness is greater than $70 \mu \mathrm{m}$, the bandgap penetration mechanism is broken and we cannot 

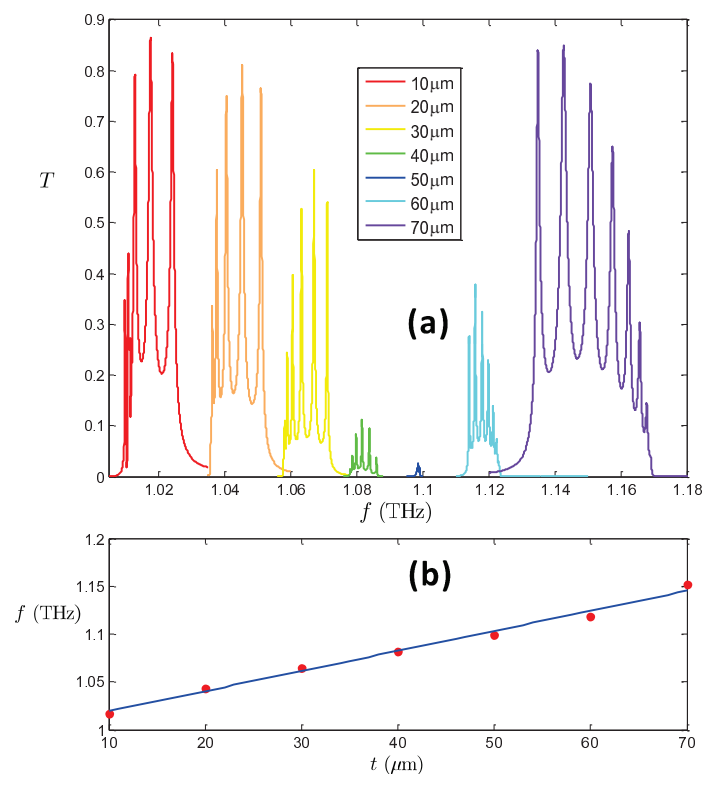

Figure 2: Transmissions and its frequency shifting for different gold thicknesses. (a) The transparencies in the stop bands presented by the lines in different colors for different gold thicknesses, respectively. (b) the center frequency of transparencies and its best fit depicted by the red bold dots and blue solid line, respectively.

find the transparency any more. So, the thicker gold layers are not included in Fig. 2 (a).

It is clear that the frequency of the transparency increases when the inner gold layer becomes thicker. The center frequency of the transparency is linearly related to the gold thickness on the whole. We can perform a least-square linear fit to the center frequency $f_{c}$ and the optimal coefficients are included in the model, in which

$$
f_{c}=0.002097 t+0.9985 \text {. }
$$

The best-fit curve and the center frequencies are also represented in Fig. 2 (b) by the solid line and the bold dots, respectively. It will be more helpful to select the gold thickness according to Eq. (3) when a specific working frequency is required. By sputtering the gold of different thicknesses, we can manipulate the transparency frequency for special $\mathrm{THz}$ waveguide devices. All transparencies are the comb functions due to the finite number of periods selected in simulations. 

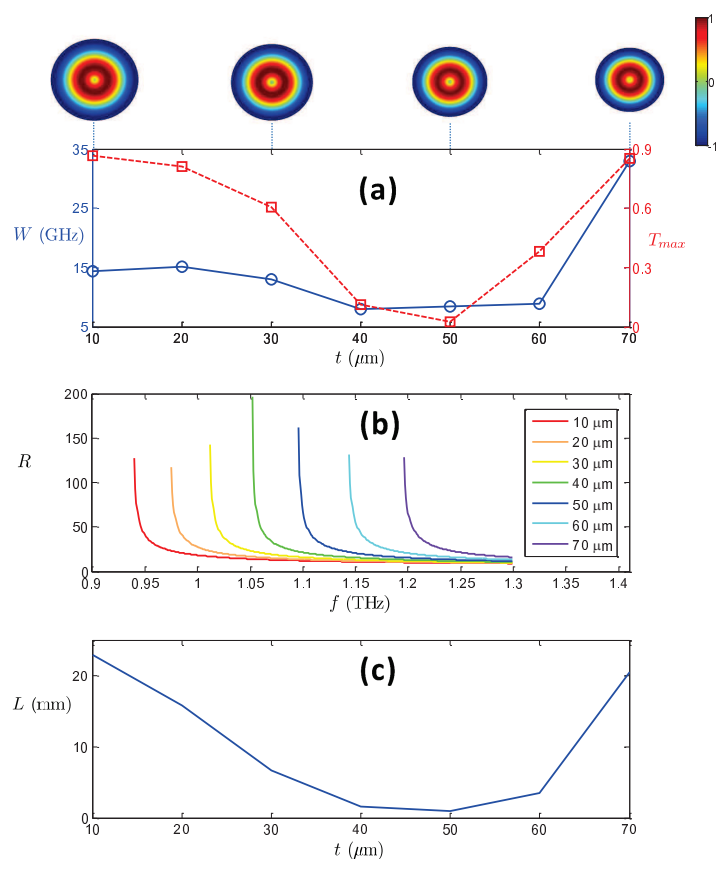

Figure 3: Transparency characteristics: (a) Band width (blue circles connected by the solid line) and maximum transmission (red squares connected by the dashed line) of the transparencies with different gold thickness. The top portion illustrates the amplitudes of the magnetic fields at the outlet, which are normalized to the maximum values of their own corresponding data series. The output patterns depict the single second modes with different mode areas. (b) Reciprocal of group velocity and its frequency manipulating by the increasing sputtered gold. (c) Propagation length for the maximum transmitted peak versus the thickness.

It is well known that the skin effect always occurs when electromagnetic waves propagate in metals. The skin depth of the gold is about tens of nanometers when the working frequency is around $1 \mathrm{THz}$. So, actually, we just reduced the effective radius of $\mathrm{THz}$ tubes when increasing the gold layer thickness. When the effective mean radius of the waveguide gets small, the working frequency goes up. By varying the sputtered gold layer, we can finally accomplish a frequency-agile single mode transparency in $\mathrm{THz}$ waveguides. 


\section{Transparency characteristics}

For different gold thicknesses, the band width $W$ and the maximum transmission (MT) $T_{\max }$ have been read out from the calculated data and are represented in Fig. 3 (a). The blue circles connected by the solid line denote the band width while the red squares connected by the dashed line depict the MT in the passband. The band width decreases gradually when the thickness increases from $10 \mu \mathrm{m}$ to $40 \mu \mathrm{m}$, but begins to increase a little until $70 \mu \mathrm{m}$. The MT reaches its minimum when the gold layer is $50 \mu \mathrm{m}$ thick. When the gold thickness is $70 \mu \mathrm{m}$, we obtain not only the maximum band width of the transparency but also the largest transmittance. The output magnitude fields, normalized to the maximum values of their own corresponding data series, are also represented in the top portion of Fig. 3 (a) for 10, 30, 50, and $70 \mu \mathrm{m}$ thick gold layers. The wave patterns are the same Bessel distributions except the mode areas indicating the single second modes transparent through the band gaps. Because of the thickened gold layers, the radius of the patterns decreases from the left to the right in the top portion of Fig. 3 (a). Thus, we have achieved not only a frequency-agile transparency but also a pure single mode of Bessel distributions.

In addition, the slow group velocity $v_{g}=2 \pi \frac{\partial f}{\partial \beta}$ has been calculated and the slow down factor $R=c / v_{g}[6,26]$ is presented in Fig. 3 (b). The different colors demonstrate the manipulation of the slow down effect by the gold thickness. The propagation length $L$ of the proposed second mode, shown in Fig. 3 (c), gets its minimum about $1 \mathrm{~mm}$ at $t=50 \mu \mathrm{m}$ in accordance with the trend of $T_{\max }$. It should be noted that the length is the order of millimeters. The reason is that the proposed waveguide mode propagates in the large air core and the effect of the excited surface plasmonic modes in the gold layers is limited. Even so, the plasmonic modes have still caused the considerable attenuation rather than the perfect metals.

According to Eq. (1), the relative position of the lines for the first and second modes in Fig. 1 (b) should be responsible for the transparency characteristics. When the gold thickness increases, the effective mean core radius decreases. The second mode of the zeroth harmonic, shown by the red parabola in Fig. 4, will move up away from the intersection of the first mode of the \pm 1 st harmonic depicted by the blue lines. At the beginning, the second mode (red parabola) moves slightly away from the intersection (circled blue point) as shown in Fig. 4 (a) and the three intersections are too close to be treated as two different interactions. The mechanism of three mode interac- 

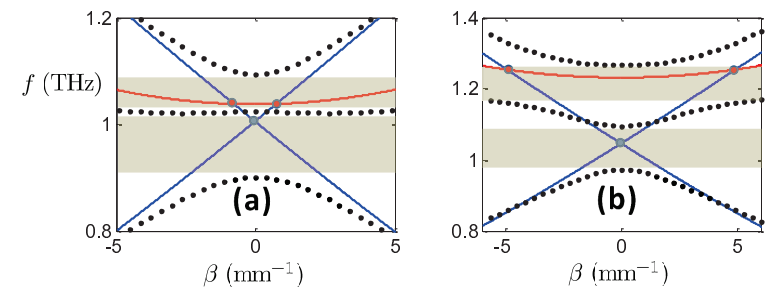

Figure 4: Multi-mode interactions with different thicknesses of gold layers. The blue and red solid lines denote the first and second modes, respectively. The circled points indicate the resonances. The bold dots constitute the related dispersion curves. The shade areas show the two bandgaps in each plot.

tions is not broken. However, the interaction becomes more intense because of the fixed height of undulation $h$. The decreasing effective mean radius results in the increasing relative corrugation amplitude, which significantly enhances the effect of resonance and enlarges the bandgaps. Therefore, the gradually increasing widths of stop bands squash the transparency. Then, the shrunk transparency is observed first when the thickness of gold layers increases. When the mean radius is small enough, the intersections (circled dots) are not very close to each other in Fig. 4 (b). The moving away resonances would not interact with each other any more and the three-modeinteraction mechanism does no longer hold. The transparency is attributed to two two-mode-resonances. Thus, the width of transparencies will increase when the second mode goes up with reduced mean radius. For the much larger thickness of gold layers, the second mode goes up higher and the upper bandgap will be covered by the pass band with hybrid modes. The single mode transparency is broken down. The bold dots shown in Fig. 4 (a) and (b) have been calculated for $t=10 \mu \mathrm{m}$ and $t=50 \mu \mathrm{m}$ with the numerical method in [24]. These results for lossless metals are not accordance with the transmissions in Fig. 2, but qualitatively interpret the trend of the band width versus the thickness. Generally, there is always a minimum transparency when the three-mode-resonance transitions into two two-mode-resonances.

\section{Conclusions}

We have proposed a single second mode transparency in a $\mathrm{THz}$ tube, which is analogous to the widely concerned EIT in transmitted spectrums. So, we have performed a classical transparency in a cylindrical tube with 
undulated gold walls. The resulting transparency, consisting of a single second mode with very narrow line-width, can be manipulated by altering the sputtered gold layers. Overall, a thicker gold layer leads to a higher working frequency. The mechanism of manipulation is attributed to the changing of inner diameters of waveguides. A transition between the three and two mode interactions is discussed in detail, which is responsible for the characteristics of transparencies. The transmitted wave patterns verified the second mode transparency in a $\mathrm{THz}$ radiation. These frequency-agile single mode transparencies (pure Bessel beams) can be efficiently coupled into a straight waveguide because of their purity and will find their applications in superluminal devices, quantum cascade lasers, nondiffractive beam producing, $\mathrm{THz}$ imaging, optical tweezers, microparticle trapping, nanofabrication, and so on. Although only a second mode transparent in a $\mathrm{THz}$ tube is proposed here, another single higher mode can also be transmitted based on the multi-mode interactions analogous to that suggested by the graph shifting method. The further work will concentrate on the realization of various single mode $\mathrm{THz}$ devices for applications.

\section{Acknowledgement}

This work was supported by the National Science Foundation of China under Grant Nos. 11074121 and 11374071, by the Fundamental Research Funds for the Central Universities of China, by the 111 project through a grant (B13015) to Harbin Engineering University, by the Opened Fund of the Key Lab of In-fiber Integrated Optics, Ministry Education of China, and by the Opened Fund of the State Key Laboratory on Integrated Optoelectronics of China under No. IOSK2013KF16.

\section{References}

\section{References}

[1] L. V. Hau, S. E. Harris, Z. Dutton, and C. H. Behroozi, "Light speed reduction to 17 metres per second in an ultracold atomic gas," Nature, vol. 397, pp. 594-598, Dec. 1999.

[2] K. Ying, Y. Niu, D. Chen, H. Cai, R. Qu, and S. Gong, "Realization of cavity linewidth narrowing via interacting dark resonances in a tripodtype electromagnetically induced transparency system," J. Opt. Soc. Am. B, vol. 31, no.1, pp. 144-148, Jan. 2014. 
[3] S. E. Harris, J. E. Field, and A. Imamoglu, "Nonlinear optical processes using electromagnetically induced transparency," Phys. Rev. Lett., vol. 64, no. 10, pp. 1107-1110, Mar. 1990.

[4] K. Totsuka, N. Kobayashi, and M. Tomita, "Slow light in coupledresonator-induced transparency," Phys. Rev. Lett., vol. 98, no. 21, pp. 213904, May 2007.

[5] N. Papasimakis, V. A. Fedotov, N. I. Zheludev, and S. L. Prosvirnin, "Metamaterial analog of electromagnetically induced transparency," Phys. Rev. Lett., vol. 101, no. 25, pp. 253903, Dec. 2008.

[6] Y. Huang, C. Min, and G. Veronis, "Subwavelength slow-light waveguides based on a plasmonic analogue of electromagnetically induced transparency," Appl. Phys. Lett., vol. 99, no. 14, pp. 143117, Oct. 2011.

[7] A. Mouadili, E. H. El Boudouti, A. Soltani, A. Talbi, A. Akjouj, and B. Djafari-Rouhani, "Theoretical and experimental evidence of Fano-like resonances in simple monomode photonic circuits," J. Appl. Phys., vol. 113, no. 16, pp. 164101, Apr. 2013.

[8] G. C. Dyer, G. R. Aizin, S. J. Allen, A. D. Grine, D. Bethke, J. L. Reno and E. A. Shaner, "Induced transparency by coupling of Tamm and defect states in tunable terahertz plasmonic crystals," Nature Photon., vol. 7, no. 11, pp. 925-930, Nov. 2013.

[9] V. A. Pogrebnyak, "Electromagnetic standing wave resonances in a periodically corrugated waveguide," Phys. Rev. E, vol. 58, no. 5, pp. R5261R5263, Nov. 1998.

[10] E. S. Lee, Y. B. Ji, and T. Jeona, "Terahertz band gap properties by using metal slits in tapered parallel-plate waveguides," Appl. Phys. Lett., vol. 97, no. 18, pp. 181112, Nov. 2010.

[11] V. Garcés-Chávez, D. McGloin, H. Melville, W. Sibbett, and K. Dholakia, "Simultaneous micromanipulation in multiple planes using a selfreconstructing light beam," Nature, vol. 419, no. 6903, pp. 145-147, Sep. 2002. 
[12] O. Brzobohatý, V. Karásek, T. C̆ižmár, and P. Zemánek, "Dynamic size tuning of multidimensional optically bound matter," Appl. Phys. Lett., vol. 99, no. 10, pp. 101105, Sep. 2011.

[13] R. E. Meyers, K. S. Deacon, A. D. Tunick, and Y. Shih, "Virtual ghost imaging through turbulence and obscurants using Bessel beam illumination," Appl. Phys. Lett., vol. 100, no. 6, pp. 061126, Feb. 2012.

[14] B. Yalizay, T. Ersoy, B. Soylu, and S. Akturk, "Fabrication of nanometer-size structures in metal thin films using femtosecond laser Bessel beams," Appl. Phys. Lett., vol. 100, no. 3, pp. 031104, Jan. 2012.

[15] M. Tonouchi, "Cutting-edge terahertz technology," Nature Photon., vol. 1, pp. 97-105, Feb. 2007.

[16] H. Bao, K. Nielsen, H. K. Rasmussen, P. U. Jepsen, and O. Bang, "Design and optimization of mechanically down-doped terahertz fiber directional couplers," Opt. Express, vol. 22, no. 8, pp. 9486-9497, Apr. 2014.

[17] O. Mitrofanov and J. A. Harrington, "Dielectric-lined cylindrical metallic THz waveguides: mode structure and dispersion" Opt. Express, vol. 18, no. 3, pp. 1898-1903, Feb. 2010.

[18] X. L. Tang, Y. Jiang, B. S. Sun, J. Chen, X. S. Zhu, P. Zhou, D. P. $\mathrm{Wu}$, and Y. W. Shi, "Elliptical hollow fiber with inner silver coating for linearly polarized terahertz transmission," IEEE Photon. Technol. Lett., vol. 25, no. 4, pp. 331-334, Feb. 2013.

[19] D. Mugnai, A. Ranfagni, and R. Ruggeri, "Observation of superluminal behaviors in wave propagation," Phys. Rev. Lett., vol. 84, no. 21, PP. 4830-4833, May 2000.

[20] M. I. Amanti, M. Fischer, G. Scalari, M. Beck, and J. Faist, "Lowdivergence single-mode terahertz quantum cascade laser," Nature Photon., vol. 3, no. 10, pp. 586-590, Sep. 2009.

[21] A. Bitman, I. Moshe, and Z. Zalevsky, "Improving depth-of field in broadband THz beams using nondiffractive Bessel beams," Opt. Lett., vol. 37, no. 19, pp. 4164-4166, Oct. 2012. 
[22] S. F. Kaijage, Z. B. Ouyang, and X. Jin, "Porous-core photonic crystal fiber for low loss terahertz wave guiding," IEEE Photon. Technol. Lett., vol. 25, no. 15, pp. 1454-1457, Aug. 2013.

[23] Z. Y. Tao, W. Y. He, and X. L. Wang, "Resonance-induced band gaps in a periodic waveguide," J. Sound Vib., vol. 313, no. 3-5, pp. 830-840, Jun. 2008.

[24] J. J. Barroso, J. P. Leite Neto, and K. G. Kostov, "Examining by the Rayleigh-Fourier method the cylindrical waveguide with axially rippled wall," IEEE T. Plasma Sci., vol. 31, no. 4, pp. 352-367, Aug. 2003.

[25] M. A. Ordal, L. L. Long, R. J. Bell, S. E. Bell, R. R. Bell, R. W. Alexander, Jr., and C. A. Ward, "Optical properties of the metals Al, $\mathrm{Co}, \mathrm{Cu}, \mathrm{Au}, \mathrm{Fe}, \mathrm{Pb}, \mathrm{Ni}, \mathrm{Pd}, \mathrm{Pt}, \mathrm{Ag}, \mathrm{Ti}$ and Win the infrared and far infrared," Appl. Opt., vol. 22, no. 7, pp. 1099-1119, April 1983.

[26] L. Yang, C. Min, and G. Veronis, "Guided subwavelength slow-light mode supported by a plasmonic waveguide system," Opt. Lett., vol. 35, no. 24 , pp. 4184-4186, Dec. 2010. 